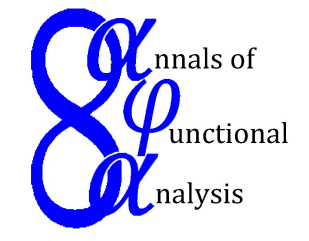

Ann. Funct. Anal. 5 (2014), no. 1, 63-69

$\mathscr{A}$ NNALS OF $\mathscr{F}$ UNCTIONAL $\mathscr{A}$ NALYSIS

ISSN: 2008-8752 (electronic)

URL:www.emis.de/journals/AFA/

\title{
2-LOCAL MAPPINGS ON ALGEBRAS WITH INVOLUTION
}

\author{
AJDA FOŠNER \\ Dedicated to Professor T. Ando with affection
}

Communicated by Z. Lykova

\begin{abstract}
We investigate 2-local *-automorphisms, 2-local *-antiautomorphisms, and 2-local Jordan *-derivations on certain algebras with involution.
\end{abstract}

\section{INTRODUCTION}

In the last few decades a lot of work has been done on local mappings of operator algebras. The main goal of the problem is to answer the question whether every local mapping of some certain class of transformations on a given algebra is global. Let us write some basic definitions and known results.

A linear mapping $\phi$ on an algebra $\mathcal{A}$ is called a local automorphism (local derivation) if for every $a \in \mathcal{A}$ there exists an automorphism (derivation) $\phi_{a}: \mathcal{A} \rightarrow \mathcal{A}$ depending on $a$ such that $\phi(a)=\phi_{a}(a)$. These two notions were introduced independently by Kadison [3] and Larson and Sourour [6]. In [3] Kadison investigated continuous local derivations on von Neumann algebras. He proved that if $\mathcal{A}$ is a von Neumann algebra and $\mathcal{M}$ a dual $\mathcal{A}$-module, then all norm-continuous local derivations from $\mathcal{A}$ into $\mathcal{M}$ are derivations. This research was motivated by problems concerning the Hochschild cohomology of operator algebras (see also [7]). On the other hand, Larson and Sourour [6, Theorem 1.2] proved that every local derivation on $\mathcal{B}(X)$, the algebra of all bounded linear operators on a Banach space $X$, is a derivation. If $X$ is infinite-dimensional, every surjective local automorphism of $\mathcal{B}(X)$ is an automorphism [6, Theorem 2.1]. Brešar and Šemrl [1] proved that the surjectivity assumption in the last

Date: Received: 23 June 2013; Accepted: 19 August 2013.

2010 Mathematics Subject Classification. Primary 47B48; Secondary 47B47.

Key words and phrases. 2-local mapping, ${ }^{*}$-automorphism, *-antiautomorphism, Jordan *derivation, algebra with involution. 
result can be removed in the case when $X$ is a separable Hilbert space. Let us also mention that, if we drop the assumption of linearity, then the corresponding statements are no longer true.

Of course, every automorphism (derivation) on an algebra $\mathcal{A}$ is a local automorphism (local derivation). But the converse is in general not true. Kadison [3] constructed an example (due to C. Jensen) of an algebra (not an operator algebra), which has nontrivial local derivations. Moreover, there are examples of local automorphisms and local derivations on operator algebras in the literature, e.g., using the subalgebra of $M_{3}(\mathbb{C})$ consisting of constant multiples of the identity plus strictly upper triangular matrices.

Let $\mathcal{A}$ be a unital Banach algebra and $\phi: \mathcal{A} \rightarrow \mathbb{C}$ a mapping having the property that $\phi(I)=1$, where $I \in \mathcal{A}$ denotes the identity. Suppose that for every $a, b \in \mathcal{A}$ there exists a multiplicative linear functional $\phi_{a, b}$ on $\mathcal{A}$ such that $\phi(a)=\phi_{a, b}(a)$ and $\phi(b)=\phi_{a, b}(b)$. Then $\phi$ is linear and multiplicative. This result was proved by Kowalski and Slodkowski in [5]. Motivated by these considerations, Šemrl [9] introduced the following definition. A mapping $\phi: \mathcal{A} \rightarrow \mathcal{A}$ is called a 2-local automorphism (2-local derivation) if for every $a, b \in \mathcal{A}$ there is an automorphism (derivation) $\phi_{a, b}: \mathcal{A} \rightarrow \mathcal{A}$ depending on $a$ and $b$ such that $\phi(a)=$ $\phi_{a, b}(a)$ and $\phi(b)=\phi_{a, b}(b)$. Let us point out that here no linearity or additivity of $\phi$ is assumed. Therefore, this notion has the advantage that it can be defined on arbitrary algebraic structures and not only on algebras. Šemrl proved that every 2-local automorphism (2-local derivation) of $\mathcal{B}(H)$, where $H$ is a separable Hilbert space, is an automorphism (derivation) [9, Theorem 1 and Theorem 2]. Our aim is to investigate $\mathrm{a}^{*}$-version of these results. First we will observe 2local *-automorphisms and 2-local *-antiautomorphisms on certain algebras with involution. After that we will study 2-local Jordan *-derivations.

\section{2-LOCAL *-AUTOMORPHISMS AND 2-LOCAL *-ANTIAUTOMORPHISMS}

Let $\mathcal{A}$ be an algebra with involution $*$. A bijective linear mapping $\phi: \mathcal{A} \rightarrow$ $\mathcal{A}$ is called a ${ }^{*}$-automorphism ( ${ }^{*}$-antiautomorphism) on $\mathcal{A}$ if $\phi(a b)=\phi(a) \phi(b)$ $(\phi(a b)=\phi(b) \phi(a))$ and $\phi\left(a^{*}\right)=\phi(a)^{*}$ for all $a, b \in \mathcal{A}$. Motivated by Šemrl's results we introduce the following definition.

Definition 2.1. A mapping $\phi: \mathcal{A} \rightarrow \mathcal{A}$ is called a 2-local *-automorphism (2local *-antiautomorphism) if for every $a, b \in \mathcal{A}$ there exists a *automorphism (*antiautomorphism) $\phi_{a, b}: \mathcal{A} \rightarrow \mathcal{A}$ depending on $a$ and $b$ such that $\phi(a)=\phi_{a, b}(a)$ and $\phi(b)=\phi_{a, b}(b)$.

Of course, every ${ }^{*}$-automorphism on a ${ }^{*}$-algebra $\mathcal{A}$ is a 2 -local ${ }^{*}$-automorphism on $\mathcal{A}$, but the converse is in general not true. Furthermore, every 2-local *automorphism is in fact a special type of 2-local automorphisms of the underlying algebra. Namely, ${ }^{*}$-automorphisms of a ${ }^{*}$-algebra $\mathcal{A}$ are automorphisms of $\mathcal{A}$ with an additional property $\phi\left(a^{*}\right)=\phi(a)^{*}, a \in \mathcal{A}$. The same is true for ${ }^{*}$ antiautomorphisms.

Remark 2.2. Note that in the definition of 2-local *-automorphisms (2-local *antiautomorphisms) we did not assumed linearity or additivity. Now, suppose 
that $\phi: \mathcal{A} \rightarrow \mathcal{A}$ is a 2-local *-automorphism (2-local *-antiautomorphisms) on a ${ }^{*}$-algebra $\mathcal{A}$. Then

$$
\phi(\lambda a)=\lambda \phi(a)
$$

for every $a \in \mathcal{A}$ and every scalar $\lambda$. Namely, there exists a *automorphism ("-antiautomorphisms) $\phi_{\lambda a, a}: \mathcal{A} \rightarrow \mathcal{A}$ such that

$$
\phi(\lambda a)=\phi_{\lambda a, a}(\lambda a)=\lambda \phi_{\lambda a, a}(a)=\lambda \phi(a) .
$$

Before continuing with our results, let us point out that every *-automorphism $\phi$ on $\mathcal{B}(H)$, the algebra of all bounded linear operators on a Hilbert space $H$, is inner, i.e., there exists a unitary $U \in \mathcal{B}(H)$ such that $\phi(A)=U A U^{*}, A \in \mathcal{B}(H)$.

The next result is a direct consequence of Theorem 1 in [9].

Theorem 2.3. Let $H$ be an infinite-dimensional separable Hilbert space and $\mathcal{B}(H)$ the algebra of all bounded linear operators on $H$. Suppose that $\phi: \mathcal{B}(H) \rightarrow \mathcal{B}(H)$ is a 2 -local ${ }^{*}$-automorphism. Then $\phi$ is $a^{*}$-automorphism on $\mathcal{B}(H)$.

Proof. Since $\phi$ is a 2-local automorphism on $\mathcal{B}(H)$, it follows from [9, Theorem 1] that $\phi$ is an automorphism on $\mathcal{B}(H)$. Moreover, there exists an invertible $T \in \mathcal{B}(H)$ such that

$$
\phi(A)=T A T^{-1}
$$

for every $A \in \mathcal{B}(H)$. Now, let $A \in \mathcal{B}(H)$. Then there exists a *automorphism $\phi_{A, A^{*}}: \mathcal{B}(H) \rightarrow \mathcal{B}(H)$ such that

$$
\phi\left(A^{*}\right)=\phi_{A, A^{*}}\left(A^{*}\right)=\phi_{A, A^{*}}(A)^{*}=\phi(A)^{*} .
$$

The proof is completed.

Remark 2.4. We actually proved that every 2-local *automorphism $\phi$ on $\mathcal{B}(H)$ is of the form

$$
\phi(A)=U A U^{*}, \quad A \in \mathcal{B}(H),
$$

where $U \in \mathcal{B}(H)$ is a fixed unitary operator.

As usual, $M_{n}(\mathbb{C})$ will denote the algebra of all $n \times n$ matrices over the field of complex numbers. In the recent paper [4] S. O. Kim and J. S. Kim proved that every 2-local *-automorphism of the algebra $M_{n}(\mathbb{C})$ is a *automorphism. In the present paper, using a different approach, we give a short proof of this result. The main idea is taken from [9].

Theorem 2.5. Every 2-local ${ }^{*}$-automorphism $\phi: M_{n}(\mathbb{C}) \rightarrow M_{n}(\mathbb{C})$ is of the form

$$
\phi(A)=U A U^{*}, \quad A \in M_{n}(\mathbb{C}),
$$

where $U \in M_{n}(\mathbb{C})$ is a fixed unitary matrix.

Proof. Let $\phi: M_{n}(\mathbb{C}) \rightarrow M_{n}(\mathbb{C})$ be a 2-local ${ }^{*}$-automorphism and let $E_{i j}, 1 \leq$ $i, j \leq n$, be the standard basis of $M_{n}(\mathbb{C})$. Denote

$$
N=\sum_{k=1}^{n-1} E_{k(k+1)} \in M_{n}(\mathbb{C}) .
$$


Since every ${ }^{*}$-automorphism of an algebra $M_{n}(\mathbb{C})$ is inner we have

$$
\phi(N)=\phi_{N, I}(N)=U N U^{*}
$$

for some unitary matrix $U \in M_{n}(\mathbb{C})$ which depends on $N$ and the identity $I \in$ $M_{n}(\mathbb{C})$. Replacing $\phi$ by the mapping $A \mapsto U^{*} A U$, if necessary, we may assume that $\phi(N)=N$. Now, let $A \in M_{n}(\mathbb{C})$. We would like to show that $\phi(A)=A$.

We know that there exists a unitary $V \in M_{n}(\mathbb{C})$ such that

$$
\phi(A)=\phi_{A, N}(A)=V A V^{*} .
$$

On the other hand,

$$
N=\phi(N)=\phi_{A, N}(N)=V N V^{*} .
$$

Therefore, $V$ commutes with $N$. Thus,

$$
V=\left[\begin{array}{cccc}
v_{1} & v_{2} & \ldots & v_{n} \\
0 & v_{1} & \ddots & \vdots \\
\vdots & \vdots & \ddots & v_{2} \\
0 & 0 & \ldots & v_{1}
\end{array}\right]
$$

for some scalars $v_{1}, v_{2}, \ldots, v_{n} \in \mathbb{C}$. Since $V$ is a unitary matrix, we have $\left|v_{1}\right|^{2}=1$ and $v_{2}=\ldots=v_{n}=0$. It follows that $V=v_{1} I$ and $\phi(A)=A$. The proof is completed.

In the same way we can actually prove that the statement holds true also for $M_{n}(\mathbb{R})$.

Remark 2.6. Let us point out that S. O. Kim and J. S. Kim [4] showed the above result using a certain preserving property. In particular, their proof heavily rely on [7, Theorem 3.4.1] which gives a non-linear characterization of the automorphisms of $M_{n}(\mathbb{C})$ via preserving the system of all eigenvalues of matrices listed according to multiplicity. Motivated by these observations, note that every 2-local *-automorphism $\phi$ on $M_{n}(\mathbb{C})$ also preserves commutativity. Namely, if $A, B \in M_{n}(\mathbb{C})$ and $A B=B A$, then there exists a *automorphism $\phi_{A, B}: M_{n}(\mathbb{C}) \rightarrow M_{n}(\mathbb{C})$ such that

$$
\begin{aligned}
\phi(A) \phi(B) & =\phi_{A, B}(A) \phi_{A, B}(B)=\phi_{A, B}(A B)=\phi_{A, B}(B A) \\
& =\phi_{A, B}(B) \phi_{A, B}(A)=\phi(B) \phi(A) .
\end{aligned}
$$

Thus, $\phi$ maps commuting pairs of matrices into commuting pairs of matrices. Therefore, we can use the results on commutativity preservers (see [10, 2, 7] and references therein) and give a new short proof of Theorem 2.5.

Now, let $\phi: \mathcal{A} \rightarrow \mathcal{A}$ be a 2-local *-antiautomorphism on a ${ }^{*}$-algebra $\mathcal{A}$ and let us define a map $\psi: \mathcal{A} \rightarrow \mathcal{A}$ by

$$
\psi(a)=\phi(a)^{*}, \quad a \in \mathcal{A} .
$$

Then it is easy to see that $\psi$ is a 2-local ${ }^{*}$-automorphism on $\mathcal{A}$. Namely, for every $a, b \in \mathcal{A}$ there exists a ${ }^{*}$-antiautomorphism $\phi_{a, b}: \mathcal{A} \rightarrow \mathcal{A}$ depending on $a$ and $b$ such that $\phi(a)=\phi_{a, b}(a)$ and $\phi(b)=\phi_{a, b}(b)$. But then the mapping $\psi_{a, b}: \mathcal{A} \rightarrow \mathcal{A}$ defined by

$$
\psi_{a, b}(c)=\phi_{a, b}(c)^{*}, \quad c \in \mathcal{A},
$$


is a ${ }^{*}$-automorphism on $\mathcal{A}$. Moreover, $\psi(a)=\psi_{a, b}(a)$ and $\psi(b)=\psi_{a, b}(b)$. Thus, we have the next two consequences of Theorem 2.3 and Theorem 2.5.

Theorem 2.7. Let $H$ be an infinite-dimensional separable Hilbert space and $\mathcal{B}(H)$ the algebra of all bounded linear operators on $H$. Suppose that $\phi: \mathcal{B}(H) \rightarrow$ $\mathcal{B}(H)$ is a 2-local ${ }^{*}$-antiautomorphism. Then $\phi$ is a ${ }^{*}$-antiautomorphism on $\mathcal{A}$. Moreover,

$$
\phi(A)=U A^{*} U^{*}, \quad A \in \mathcal{B}(H),
$$

where $U \in \mathcal{B}(H)$ is a fixed unitary operator.

Theorem 2.8. Every 2-local ${ }^{*}$-antiautomorphism $\phi: M_{n}(\mathbb{C}) \rightarrow M_{n}(\mathbb{C})$ is of the form

$$
\phi(A)=U A^{*} U^{*}, \quad A \in \mathcal{A},
$$

where $U \in M_{n}(\mathbb{C})$ is a fixed matrix.

\section{2-LOCAL JORDAN * -DERIVATIONS}

Let $\mathcal{A}$ be a complex algebra with involution $*$. A real linear mapping $\delta: \mathcal{A} \rightarrow \mathcal{A}$ is called a Jordan *-derivation on $\mathcal{A}$ if $\delta\left(a^{2}\right)=\delta(a) a^{*}+a \delta(a)$ for every $a \in \mathcal{A}$. Analogously, as Šemrl introduced 2-local derivations, we can define 2-local Jordan *-derivations.

Definition 3.1. A mapping $\delta: \mathcal{A} \rightarrow \mathcal{A}$ is called a 2-local Jordan *-derivation if for every $a, b \in \mathcal{A}$ there exists a Jordan ${ }^{*}$-derivation $\delta_{a, b}: \mathcal{A} \rightarrow \mathcal{A}$ depending on $a$ and $b$ such that $\delta(a)=\delta_{a, b}(a)$ and $\delta(b)=\delta_{a, b}(b)$.

Remark 3.2. In the above definition we did not assumed linearity or additivity of $\delta$. But, in the same way as in Remark 2.2, we can show that

$$
\delta(\lambda a)=\lambda \delta(a)
$$

for all $a \in \mathcal{A}$ and every scalar $\lambda \in \mathbb{R}$. Moreover, for every $a \in \mathcal{A}$ there exists an appropriate Jordan ${ }^{*}$-derivation $\delta_{a^{2}, a}: \mathcal{A} \rightarrow \mathcal{A}$ such that

$$
\delta\left(a^{2}\right)=\delta_{a^{2}, a}\left(a^{2}\right)=\delta_{a^{2}, a}(a) a^{*}+a \delta_{a^{2}, a}(a)=\delta(a) a^{*}+a \delta(a) .
$$

Thus,

$$
\delta\left(a^{2}\right)=\delta(a) a^{*}+a \delta(a)
$$

for all $a \in \mathcal{A}$.

Of course, every Jordan *-derivation on a *-algebra $\mathcal{A}$ is a 2-local Jordan *derivation on $\mathcal{A}$. But in general we can not expect the converse statement. And the natural question here is, for which *-algebras every 2-local Jordan *-derivation is automatically a Jordan *-derivation.

Let $H$ be a complex Hilbert space, $\operatorname{dim} H>1$, and $\mathcal{B}(H)$ the algebra of all bounded linear operators on $H$. We will denote by $\mathcal{F}(H) \subseteq \mathcal{B}(H)$ the subalgebra of bounded finite rank operators. We call a subalgebra $\mathcal{A}$ of $\mathcal{B}(H)$ standard, if it contains $\mathcal{F}(H)$. Šemrl [8] proved that every Jordan ${ }^{*}$-derivation $\delta: \mathcal{A} \rightarrow \mathcal{B}(H)$ is of the form $\delta(A)=T A^{*}-A T, A \in \mathcal{A}$, where $T \in \mathcal{B}(H)$ is a fixed operator. 
Theorem 3.3. Let $H$ be a complex Hilbert space, $\operatorname{dim} H>1$, and $\mathcal{A}$ a unital standard operator algebra on $H$. Suppose that $\delta: \mathcal{A} \rightarrow \mathcal{B}(H)$ is a 2-local Jordan ${ }^{*}$-derivation. Then $\delta$ is a Jordan ${ }^{*}$-derivation on $\mathcal{A}$. Moreover, there exists a linear operator $T \in \mathcal{B}(H)$ such that

$$
\delta(A)=T A^{*}-A T
$$

for every $A \in \mathcal{A}$.

Proof. Let $\delta: \mathcal{A} \rightarrow \mathcal{B}(H)$ be a 2-local Jordan ${ }^{*}$-derivation on $\mathcal{A}$. Without loss of generality we may assume that $\delta(i I)=0$. Namely, if $\delta(i I) \neq 0$, we replace a mapping $\delta$ with a mapping $\delta-\delta_{i I, I}$, where $\delta_{i I, I}$ is an appropriate Jordan *derivation depending on $i I$ and the identity $I \in \mathcal{A}$. Now, let $A \in \mathcal{A}$. We would like to prove that $\delta(A)=0$.

We have

$$
\delta(A)=\delta_{A, i I}(A)=T A^{*}-A T
$$

for some $T \in \mathcal{B}(H)$ depending on $A$ and $i I$. On the other hand,

$$
\delta(i I)=\delta_{A, i I}(i I)=T(i I)^{*}-(i I) T=-2 i T=0 .
$$

This yields that $T=0$ and $\delta(A)=0$. Thus, $\delta$ is a Jordan *-derivation on $\mathcal{A}$ and, according to [8, Theorem], there exists $T \in \mathcal{B}(H)$ such that

$$
\delta(A)=T A^{*}-A T
$$

for every $A \in \mathcal{A}$. The proof is completed.

Theorem 3.4. Every 2-local Jordan ${ }^{*}$-derivation $\delta: M_{n}(\mathbb{C}) \rightarrow M_{n}(\mathbb{C})$ is of the form

$$
\delta(A)=T A^{*}-A T, \quad A \in \mathcal{A},
$$

where $T \in M_{n}(\mathbb{C})$ is a fixed matrix.

Proof. The main idea of the proof is the same as the main idea in the proof of Theorem 3.3. First we assume that $\delta(i I)=0$, where $I \in M_{n}(\mathbb{C})$ is the identity matrix. Then we take an arbitrary matrix $A \in M_{n}(\mathbb{C})$ and show that $\delta(A)=0$, which yields that $\delta$ is a Jordan ${ }^{*}$-derivation on $M_{n}(\mathbb{C})$. Moreover, there exists a matrix $T \in M_{n}(\mathbb{C})$ such that $\delta(A)=T A^{*}-A T$ for every $A \in M_{n}(\mathbb{C})$.

Acknowledgement. This paper is dedicated to Professor Tsuyoshi Ando for his significant contributions to several areas of linear algebra, operator theory, and functional analysis.

\section{REFERENCES}

1. M. Brešar and P. Šemrl, On local automorphisms and mappings that preserve idempotents, Studia Math. 113 (1995), 101-108.

2. A. Fošner, Commutativity preserving maps on $M_{n}(\mathbb{R})$, Glasnik Mat. 44 (2009), 127-140.

3. R.V. Kadison, Local derivations, J. Algebra 130 (1990), 494-509.

4. S.O. Kim and J.S. Kim, Local automorphisms and derivations on $M_{n}$, Proc. Amer. Math. Soc. 132 (2003), 1389-1392.

5. S. Kowalski and Z. Slodkowski, A characterization of multiplicative linear functionals in Banach algebras, Studia Math. 67 (1980), 215-223. 
6. D. Larson and A.R. Sourour, Local derivations and local automorphisms of $\mathcal{B}(X)$, Proc. Symp. Pure Math. 51 (1990), 187-194.

7. L. Molnár, Selected preserver problems on algebraic structures of linear operators and on function spaces, Lecture Notes in Mathematics, Vol. 1895, Springer, 2007.

8. P. Šemrl, Jordan ${ }^{*}$-derivations on standard operator algebras, Proc. Amer. Math. Soc. 120 (1994), 515-518.

9. P. Šmrl, Local automorphisms and derivations on $\mathcal{B}(H)$, Proc. Amer. Math. Soc. 125 (1997), 2677-2680.

10. P. Šemrl, Commutativity preserving maps, Linear Algebra Appl. 429 (2008), 1051-1070.

Faculty of Management, University of Primorska, Cankarjeva 5, Si-6104 Koper, Slovenia.

E-mail address: ajda.fosner@fm-kp.si 